3. In anchylosis after traumatic inflammation in healthy adults, such as that which occurs after wounds of the joints, and gun-shot wounds in the neighbourhood of the joints, when the joint itself has escaped injury; and in some cases of anchylosis, chiefly from long-retained position, occurs, as a general rule, no destruction of bone even after acute suppurative inflammation, the soft tissues only being involved.

4. In anchylosis after strumous disease of the joint, when arrested in the early stage, without the occurrence of suppuration, or, at least, of abscess bursting externally, there is generally only a superficial caries of the head of the bone; and the destruction being thus limited in extent, the neck of the thigh-bone remains of its natural length, although practically somewhat shortened by being depressed, or sunk into the acetabulum. In this class of cases, however, the operation can generally be performed.

5. In anchylosis following the more severe forms of strumous disease, in which there has been evidence of caries and necrosis of bone, with abscesses bursting externally, and remaining open a considerable time, generally giving exit to small particles of bone, destruction of the head and neck of the thigh-bone, to a greater or less extent, may be diagnosed; and in all such cases, the operation cannot be performed.

Thus it will be seen that, out of the five classes of bony anchylosis above described, in three classes the head and neck of the thigh-bone remain of their full natural proportions. In the fourth class, although some difficulty may occasionally be met with, the operation can generally be performed; and it is only in the fifth class of cases that the operation is decidedly negatived.

\section{A CASE OF POISONING BY NITRATE OF SILVER.}

By THOMAS SCATTERGOOD, EsQ.,

Lecturer on Forensic Medicine at the Leeds School of Medicine.

PoIsoning by nitrate of silver is of rare occurrence. It is not mentioned by Casper, Tardieu ("Étude"), nor Wormley. Orfila describes the symptoms, but mentions no case. Beck refers to two cases : one related by Metzger, in which the most imminent danger followed from a surgeon letting a piece drop into the throat of a patient; the other taken from Boerhaave, which is also alluded to in Pereira's Materia Medica. Dr. A. S. Taylor, in his work on Poisons, states that there are at least two cases recorded, and that one of them, the particulars of which are unknown, occurred in 1837.8 ; but he gives no references. It seems, therefore, desirable to place on record the following case, which occurred in February of the present year.

A medical pupil, while touching a small ulcer beneath the tongue of a child aged 15 months, with a stick of nitrate of silver three-quarters of an inch long, which he held in his fingers by one end wrapped in paper, had the misfortune to let it slip down the child's throat. The following notes of the symptoms were kindly furnished to me by the practitioner in whose surgery the occurrence took place, and who himself saw the child immediately after it. "Almost immediately the child vomited some oily matter, supposed to be cod-liver oil and milk, the oil having been taken shortly after breakfast. When the vomiting had ceased, and within a few minutes of the caustic being swallowed, common salt was given in considerable quantity, after which the child was slightly convulsed. Again vomiting took place, and now the matter ejected had a white curdy appearance, and no doubt was principally chloride of silver. The administration of salt was repeated frequently. Vomiting and convulsions occurred at short intervals till I I.30." [The caustic had been swallowed at 9.30.] "At that time there was a copious liquid stool, containing a quantity of the white curdy substance. At I P.M., the child fell into a composed sleep, which lasted for half an hour ; after which convulsions returned with increased violence, and continued until 3. The extremities then became cold, the face pinched, the skin clammy, and the pulse almost imperceptible; and child died in violent convulsions at 330 P.M., six hours after swallowing the poison."

By the direction of the coroner, I made a post mortem examination, which took place twenty-five hours and a half after death.

The child was well nourished, and appeared to have been in good health ; cadaveric rigidity was well marked ; the expression of countenance was placid. Under the tongue was a small hard swelling, ulcerated on the surface, corresponding to the orifice of the Whartonian duct. There were no marks of nitrate of silver in the mouth, but in the esophagus two or three small patches of corrosion. The stomach contained two ounces and a half of inodorous fluid. Its mucous membrane was pale in colour, except one small point of ecchymosis, and exhibited a large patch of corrosion, interrupted transversely in several places, but extending from the cardiac opening along the greater curvature for four inches. It was half an inch wide at the cardiac end, and one and a half inches at the end nearest the pylorus, and was of a brilliant white colour. In the duodenum, and the first twelve inches of the jejunum, the valvulæ conniventes presented a similar corrosion over nearly their whole surface, but of a greyer colour. This was not washed off by a gentle stream of water, nor by rubbing with the finger, by which, however, loose white particles were removed. There were no morbid appearances in the other parts of the alimentary canal, in which about three ounces of fluid were contained. The other viscera were healthy. The heart was empty and contracted. The mucous membrane of the trachea was normal. The contents of the stomach, ilium, and rectum, were separately analysed. In each there was a considerable quantity of chloride of sodium, most in the stomach, and least in the rectum; and of course, therefore, in none of them was there any nitrate of silver. The white particles rubbed off the corroded patches in the stomach, and off the valvulæ conniventes, were proved to be chloride of silver.

It appeared that, although the antidote was administered promptly and in sufficient excess to neutralise the whole of the poison, yet probably much mischief had already been done before the administration commenced; and the solid stick of nitrate of silver, retained by its weight in contact with the most depending part of the stomach, continued to destroy its mucous membrane even while its own upper surface was undergoing decomposition by the chloride of sodium; and meanwhile a sufficient quantity of strong solution of the caustic flowed through the pyloric orifice, to damage the mucous surface of a considerable part of the small intestine before it was reached by the chloride. I think that the free administration of albumen or of milk should be combined in such a case with the use of the chemical antidote.

Since writing the above, my attention has been called to a case of poisoning by solution of nitrate of silver related by Devergie (Médecine Ligale, vol. iii) on the authority of $\mathbf{M}$. Pommarede. The quantity taken was uncertain; the patient estimated it at 8 grammes (an ounce). The patient was twenty-one years of age. On his admission to hospital, there were insensibility, convulsive movements of the upper limbs and face, forcible closure of the jaw, dilatation and insensibility of the pupils ; pulse 70, full and natural. The treatment consisted in the administration of solution of common salt at intervals of a quarter of an hour. Under this treatment, he improved; but the next day the symptoms reappeared, and again improved in the evening, when, after taking some barley-water, he vomited abundantly. In a few days, he was discharged well, having for several days suffered from pains in the epigastrium, which, however, were never very intense.

\section{RECOLLECTIONS OF THE MEDICAL SCHOOL OF BERLIN.}

By H. ROSBOROUGH SWANZY, M.B. Dub.,

Ophthalmic Surgeon to the Adelaide Hospital; late Assistant at the late Professor von Graefe's Hospital, Berlin.

As a pendant to Dr. Payne's interesting papers on the Medical School of Vienna, the present contribution may be acceptable to some of the readers of the JOURNAL.

From experience, I am ready to endorse all that Dr. Payne has advanced in favour of Vienna as a place of study for the English student, but fear that some may have been led, by his glowing descriptions, to think that it is the only place worth going to, and the place for everyone to go to. The Vienna School has, indeed, the great charm of being eminently practical ; but, when the British student has completed his term of professional education at home, and obtained his diploma, he is, as a general rule, as well versed in practical clinical medicine and surgery as it is possible he should be in the time, with all the advantages of good teachers and abundant opportunities at hand. I imagine, therefore, that when he then visits a foreign school, his primary object is not to obtain additional practical knowledge of these branches-for this might be attained by a longer course of study at home-but rather to gain new modes of thinking on facts already secured, and to study those branches of the profession for which he has had imperfect or no advantages in England.

Berlin supplies these desiderata in some subjects better, in others equally well, and, again, in others not so well as Vienna. The student should know what he wants, and go where he can get it best.

In the first place, with regard to clinical medicine : the two great teachers of this subject in Berlin, are Professors Frerichs and Traube. The clinique of the former is probably the best attended. It is conducted in one of the theatres of the Charité, three or four times a week, 